\title{
The Early Outcome Of the Bernese Periacetabular Osteotomy For Hip Dysplasia In Multiple Epiphyseal Dysplasia
}

\section{Yao-Yuan Chang}

National Taiwan University Hospital

Chia-Che Lee

National Taiwan University Hospital

Sheng-Chieh Lin

Chung Shan Medical University Hospital

Ken N Kuo

National Taiwan University Hospital

Kuan-Wen Wu

National Taiwan University Hospital

Ting-Ming Wang ( $\nabla$ dtorth76@yahoo.com.tw)

National Taiwan University Hospital

\section{Research Article}

Keywords: Multiple epiphyseal dysplasia (MED) , Harris Hip Score (HHS)

Posted Date: July 16th, 2021

DOI: https://doi.org/10.21203/rs.3.rs-691751/v1

License: (c) (i) This work is licensed under a Creative Commons Attribution 4.0 International License.

Read Full License 


\section{Abstract}

\section{Background:}

Multiple epiphyseal dysplasia (MED) is a rare congenital bone dysplasia. Patients with MED develop secondary hip osteoarthritis as early as third to the fourth decade. Currently, there is no consensus on how to prevent or slow the process of secondary hip osteoarthritis.

The Bernese periacetabular osteotomy is a joint preserving surgery to reshape acetabulum and extend coverage for the hip, however, there is no established evidence of the effectiveness for the MED hips.

\section{Patients and methods:}

A retrospective series of 6 hips in 3 patients with multiple epiphyseal dysplasia treated with the Bernese periacetabular osteotomy were reviewed. The average age at the time of surgery was 14.3 years (range: 11.4 to $17.2 \mathrm{y}$ ). Radiographic parameters were analyzed preoperatively and 1-year postoperatively. The hip function was evaluated by the Harris Hip Score (HHS) before and after surgery.

\section{Results:}

The mean follow-up time was 1.7 years. The mean LCEA increased from $3.8^{\circ}$ to $47.1^{\circ}(p=.02)$, ACEA increased from $7.3^{\circ}$ to $35.1^{\circ}(p=.02)$, and Al decreased from $27.8^{\circ}$ to $14.6^{\circ}(p=.04)$. The femoral head coverage ratio increased from $66.8 \%$ to $100 \%(p=.02)$. The procedure achieved femoral head medialization by decreasing central head distance from $86.7 \mathrm{~mm}$ preoperatively to $82.7 \mathrm{~mm}$ postoperatively, however, without statistical significance. $(p=.699)$. The improvement of clinical outcomes by mean HHS was significant from 67.3 preoperatively to 86.7 postoperatively $(p=0.05)$.

\section{Conclusion:}

Bernese PAO is a feasible option for treatment of the hip problems in MED patients. It reshapes acetabular and femoral morphology 3-dimensionally. In our study, the short-term follow-up results showed obvious functional and radiographic improvement. A long-term follow-up is necessary in the future.

\section{Introduction}

Multiple epiphyseal dysplasia (MED) is a rare, congenital bone dysplasia characterized by abnormal ossification of multiple epiphyses. ${ }^{1}$ It affects 10 per 100,000 individuals worldwide. ${ }^{2}$ The clinical presentations include short stature, angular or rotational deformity of the extremities, intermittent joint pain, and/or precocious osteoarthritis. Weight-bearing joints are easily to be affected especially for hip joints. ${ }^{3}$ Mäkitie et al. showed all patients with MED had abnormal development of the proximal femoral epiphyses before skeletal maturity in a 12 patients study. ${ }^{4}$ Patients with MED would face secondary hip osteoarthritis as early as the third to the fourth decade. The information on the results of total hip 
arthroplasty in patients with MED was limited. Ramaswamy R et al. reported 16 hips with MED and secondary osteoarthritis received total hip arthroplasty. The mean age at surgery was in the third to fourth decade of life. Ten hips required revision at a mean of 12.5 years. ${ }^{3}$ To prevent secondary hip osteoarthritis, the possible treatments include conservative treatments (reduced weight bearing with assistive device, control body weight and physical therapy to increase muscular strength) and acetabular osteotomy. Chiari Osteotomy, Steel triple innominate osteotomy and Dega osteotomy have been reported to treat patient with MED in different age to redirect or reshape the acetabulum. The short-term results were satisfactory. Currently, there is no consensus on how to prevent secondary hip osteoarthritis. ${ }^{1}$

The Bernese periacetabular osteotomy (PAO) is a technique to reshape acetabulum and extend coverage for hip. The most frequent surgical indication is symptomatic acetabular dysplasia in adolescents or young adults with correctable deformity and preserved range of motion. The contraindications include advanced osteoarthritis and irreducible incongruity of the hip joint. ${ }^{5}$ Currently, the Bernese PAO is recognized as an effective surgery to prevent secondary hip osteoarthritis in patients with hip dysplasia. The 10-year cumulative hip survivorship is $80-90 \% .{ }^{6}$ Comparing to other periacetabular osteotomy, with intact posterior column, the Bernese PAO provides better stability. In patients with MED, although there are acetabular dysplasia and hip joint incongruity, however, most of hips joint can be reduced with hip joint in internal rotation, flexion, and abduction. In our hypothesis, these hips can be savaged with Bernese PAO.

The objectives of the present study are to assess outcomes in patients with Multiple epiphyseal dysplasia undergoing Ganz osteotomy.

\section{Materials And Methods}

\section{Study participants and surgical procedure}

This retrospective study includes consecutive patients with diagnosis of multiple epiphyseal dysplasia between January 2015 and December 2020. The diagnosis was based on clinical presentations and plain radiographs by pediatric geneticists and orthopaedic surgeons. The preoperative and postoperative clinic records and radiographs were available for the study. All patients received Bernese PAO with minimum of one-year follow-up.

A total of 6 hips in 3 patients with multiple epiphyseal dysplasia fulfill the criteria of inclusion. Indications for surgery included limp, a minimum of conservative treatment of intolerable hip pain for more than 6 months, lateral center-edge angle (LCEA) less than 20 degrees and Tonnis grade less than 2. All patients had 3D-CT scan of the hips before surgery to check the congruity and coverage of the hip joint. Only reducible hip joints without hinge abduction in frog leg radiographs met the criteria for surgery.

The surgical incision was performed via Smith-Petersen incision about 6-8 $\mathrm{cm}$ which is smaller than the original described. Osteotomies of the anterior portion of ischium, the superior pubic ramus, the ilium, and then along the posterior column are performed via angled bone chisel. After osteotomies, the fragments 
are fixed with threaded pins, or cannulated screws. At the end of surgery, all patients are checked for hip internal rotation of 20 degrees with hip flexion to 90 degrees without difficulty.

\section{Post-operative Protocol}

After operation, early rehabilitation program was started. With physical therapist's assistance, the patients started toe touch bearing ambulation. Weight bearing as tolerated with crutch assistance started 1-month postoperatively. Then the patients would gradually advance to full weight bearing with no restriction 6months postoperatively and eventually returned to normal full daily activity.

\section{Imaging Evaluation}

Radiographic parameters were analyzed preoperatively and 1-year postoperatively. The radiographic parameters included femoral head coverage ratio, lateral center-edge angle (LCEA), acetabulum index (AI), central head distance, and Shenton's line on the AP view. Anterior center-edge angles (ACEA) were recorded on false profile view. The leg length was measured on the scanogram.

\section{Clinical Evaluation}

Hip function was evaluated by the Harris Hip Score (HHS) before surgery and 1-year postoperatively.

\section{Statistical analysis}

Pre- and postoperative radiographic parameters and HHS scores were compared using a Mann-Whitney U test. Statistically significance level was at $p<0.05$. The statistical analysis was performed by SPSS software system.

\section{Ethical approval}

All procedures performed in studies involving human participants were in accordance with the ethical standards of the National Taiwan University Hospital Research Ethics Committee.

\section{Informed consent}

In this study, participants under the age of 18 years, informed consent was obtained from a parent and/or legal guardian.

\section{Statement}

The National Taiwan University Hospital Research Ethics Committee has approved the study (NTUH-REC No. 202105093RINC). 


\section{Results}

\section{Demographics}

In our institution, there were 3 female patients with multiple epiphyseal dysplasia who had minimum of I year follow-up following surgery. Triradiate cartilages were closed in all plain pelvis radiographs. All 3 patients received bilateral Ganz osteotomy in stages. None of them received other surgical interventions before Ganz osteotomy. The mean age was 14.3 years (range from 11.4 to 17.2) and the mean body mass index was 20.3. The mean follow-up time was 1.7 years (Table 1 ).

Table 1. Demographics

\begin{tabular}{|lllll|}
\hline Patient No. & Side & Age & Major complication & Follow-up (y) \\
\hline 1 & Right & 15.6 & Nil & 1.21 \\
\hline 1 & Left & 15.0 & Nil & 1.81 \\
\hline 2 & Right & 11.4 & Nil & 2.38 \\
\hline 2 & Left & 10.8 & Nil & 2.98 \\
\hline 3 & Right & 17.2 & Nil & 1.51 \\
\hline 3 & Left & 16.6 & Nil & 2.11 \\
\hline
\end{tabular}

\section{Radiographic results}

Preoperative 3D-CT showed all 3 patients had incongruent hip joint surface and global dysplasia at acetabulum with variable deficiencies in coverage were also observed.

The mean LCEA increased from 3.8 to $47.1(p=.02)$, ACEA increased from 7.3 to $35.1(p=.02)$, and Al decreased from 27.8 to $14.6(p=.04)$. The femoral head coverage ratio increased from $66.8 \%$ to $100 \%$ ( $p=$ .02). Femoral head medialization was revealed by decreased central head distance from $86.7 \mathrm{~mm}$ to $82.7 \mathrm{~mm}$ without statistical significance. $(p=.699)$. The leg length had no obvious difference after surgery. All broken Shenton lines restored following surgery (Table 2). An example of the patient No.2 radiographs is shown as Figure 1.

All osteotomies achieved bony union at 6 months following surgery. There was no delayed union or nonunion observed. No progression of hip osteoarthritis change was noted during follow-up. All preoperative and postoperative radiographs were Tönnis grade less than 2 .

Table 2. Radiographic Parameter 


\begin{tabular}{|llllll|}
\hline \multirow{2}{*}{ parameter } & \multicolumn{2}{l}{ Pre-operation } & \multicolumn{2}{l}{ Post-operation } & \multirow{2}{*}{ p-value } \\
\cline { 2 - 5 } & Mean & Range & Mean & Range & \\
\hline LCEA (deg.) & 3.8 & -23.1 to 14.4 & 47.1 & 37.7 to 55.4 & 0.02 \\
\hline ACEA (deg.) & 7.3 & -8.3 to 16.2 & 35.1 & 29.9 to 41.0 & 0.02 \\
\hline Al (deg.) & 27.8 & 24.9 to 31.8 & 14.6 & 5.8 to 21 & 0.04 \\
\hline Femoral head coverage (\%) & 66.8 & 46 to 79 & 100 & 100 to 100 & 0.02 \\
\hline Central head distance (mm) & 86.7 & 83 to 91 & 82.7 & 70 to 88 & 0.699 \\
\hline Leg length (cm) & 63.5 & 56.5 to 69.6 & 62.8 & 53.5 to 69.7 & 0.463 \\
\hline Shenton line & Broken & \multicolumn{5}{c}{ smooth } \\
\hline
\end{tabular}

\section{Clinical results}

The hip pain and function were all improved after operation. The improvement of clinical outcomes by mean HHS was significant from 67.3 preoperatively to 86.7 postoperatively $(p=0.05)$.

\section{Complications and Estimated Blood Loss}

There was no obvious complications including massive bleeding, surgical site infection, nerve palsy or nonunion observed during clinic follow-up. Post-operative course was smooth and all patients recovered well physically. There was no conversion to total hip replacement.

\section{Discussion}

Multiple epiphyseal dysplasia is caused by abnormalities in type IX collagen and cartilage oligomeric matrix protein. ${ }^{7}$ Most of patients develop deformities of the extremities during childhood. The symptoms including pain and deformities of lower extremity that can be noted as early as 2-year-old. ${ }^{8}$ Due to disorganized endochondral ossification of the epiphyses of long bones, the articular cartilage lacks underlying osseous support that results in degeneration. Cartilage degeneration is most prominent in weight-bearing joints, especially hip joint. ${ }^{9}$ End-stage hip secondary osteoarthritis at a relatively young age is common. However, current results of total hip replacement in patients with MED was discontented.

There is no clear treatment guideline to prevent secondary hip osteoarthritis. At present, many studies indicate that it is progressive and suggests only conservative treatment. In Kim et al. study of 40 patients with MED involved hip joints treated with conservative treatment including limited weight bearing, control of body weight, physical therapy. The results revealed less severity of the hip deformity and improved hip function. ${ }^{1}$ Nevertheless, the conservative treatment only delays osteoarthritis progression and the 
improvement is time-consuming. More importantly, the daily activity can be restricted in order to reduce the burden of the joint. It does not correct the deformity and femoral head morphology.

Patients with MED may have a wide spectrum of hip joint deformity. Surgical intervention including proximal osteotomy or periacetabular osteotomy could be considered as a treatment of choice for these patients. For patients with varus or valgus deformity combined with hip dysplasia, proximal femoral osteotomy can be a suitable treatment. ${ }^{10}$ Lian et al. reported 2 patients with MED and received intertrochanteric extension osteotomy. Joint function, coxa vara deformity and femoral head coverage were improved in mid-term follow-up. ${ }^{11}$ However, for those patients with inadequate coverage of the femoral head and acetabular side change, a rotational osteotomy of the pelvis would be superior to intertrochanteric osteotomy. ${ }^{12}$ Periacetabular osteotomy (PAO) is still the gold-standard treatment for hip dysplasia. Wyles et al. revealed PAO can unequivocally improve the natural history in patients with hip dysplasia. ${ }^{13}$ Although surgical intervention could not alter the ossification of the epiphysis, we believed that an acetabular correction would improve the prognosis of native hip. Sponer et al. reported 12 hips in 11 patients with MED were treated by the Steel triple innominate osteotomy. The mean follow-up time was 2 years with the correction of LCEA angle. ${ }^{14}$ However, other radiographic results were not reported. In our study, LCEA, ACEA, Al and femoral head coverage were all improved after operation. We demonstrated that Bernese PAO can correct acetabular and femoral morphology 3-dimensionally. Lian et al. reported 2 patients with MED and severe hip deformity were treated with Dega osteotomy. The mid-term outcomes including functional and radiographic were good. ${ }^{11}$ However, it still needs long-term follow-up and larger sample size to prove the efficacy of the surgery. We believed Bernese PAO has some advantages that Steel triple innominate osteotomy and Dega osteotomy cannot achieve. On the one hand, those osteotomies and bone graft placement are relatively unstable. Post-operation immobilization would be required for a much longer time. On the other hand, it is difficult to achieve optimal medialization and coverage of the acetabulum. ${ }^{15}$

Among periacetabular osteotomy, Bernese PAO is a novel technique and has advantages of better stability, maximal mobility, preservation of the acetabular blood supply, preservation of the hip abductor musculature, medialization of acetabulum and powerful deformity correction. Furthermore, it could combine with adjunctive procedures if other hip deformity exists. ${ }^{5} \mathrm{~A}$ recent study pointed out Bernese PAO has provided a satisfactory surgical approach in the treatment of the hip with global dysplasia in cerebral palsy patients. ${ }^{16}$ With global dysplasia of the acetabulum in our patients, we believe that Bernese PAO is a powerful armamentarium to correct this deformity.

In our studies, the mean age of patients was 14.3 years. Plain films also revealed triradiate cartilages were closed. For hip dysplasia, Bernese PAO is the treatment of choice in our hands for those patients, especially, for global dysplasia of the acetabulum evident from preoperative 3D-CT scan. To the best of our knowledge, this is the first study using Bernese PAO to treat MED patient with hip dysplasia. The short-term outcome is satisfactory. Radiographic parameter including LCEA, ACEA, Al and the femoral head coverage ratio were significantly improved. Furthermore, hip function had corresponding progress. 
The patient could have normal activity without restriction. No major complication was noted during surgery or follow-up. For the small incision we carried out, the wound pain could be better tolerated under medication and the patient could start rehabilitation as early as 1-day post operation. Furthermore, the Bernese PAO provides adequate medialization of hip joint. In case the osteoarthritis progresses, a better joint morphology can offer easier positioning of total hip replacement.

There are limitations to our study. First of all, the case number is small and retrospective. However, due to the low incidence of MED, it is hard to perform a large sample and prospective study. Second, it is a short follow-up. Our study still provides valuable information for the clinicians who are involved in management of skeletal dysplasia population. A longer study is necessary to understand the natural history of the operated hips.

\section{Conclusions}

Bernese PAO is a feasible option for treatment of the hip problems in MED patients. It reshapes acetabular and femoral morphology 3-dimensionally. In our study, the short-term follow-up results showed obvious functional and radiographic improvement. A long-term follow-up is desirable in the future.

\section{References}

1. Kim, S. J., Ramanathan, A. K., Jeon, Y. S. \& Song, H. R. The fate of hips that are conservatively treated in multiple epiphyseal dysplasia. J Pediatr Orthop B. Nov 2017;26(6):526-531.

2. Vanlommel, J., Vanlommel, L., Molenaers, B. \& Simon, J. P. Hybrid total hip arthroplasty for multiple epiphyseal dysplasia. Orthopaedics \& Traumatology: Surgery \& Research. 2018;104(3):301-305.

3. Ramaswamy, R., Kosashvili, Y. \& Cameron, H. Total hip replacement in patients with multiple epiphyseal dysplasia with a mean follow-up of 15 years and survival analysis. J Bone Joint Surg Br. Apr 2010;92(4):489-495.

4. Mäkitie, O. et al. Clinical and radiographic findings in multiple epiphyseal dysplasia caused by MATN3 mutations: description of 12 patients. American journal of medical genetics. Part A. Mar 15 2004;125a(3):278-284.

5. Kamath, A. F. Bernese periacetabular osteotomy for hip dysplasia: Surgical technique and indications. World J Orthop. May 18 2016;7(5):280-286.

6. Lerch, T. D., Steppacher, S. D., Liechti, E. F., Siebenrock, K. A. \& Tannast, M. [Bernese periacetabular osteotomy.: Indications, technique and results 30 years after the first description]. Orthopade. Aug 2016;45(8):687-694.

7. Mabuchi, A. et al. Novel and recurrent mutations clustered in the von Willebrand factor A domain of MATN3 in multiple epiphyseal dysplasia. Hum Mutat. Nov 2004;24(5):439-440.

8. Miura, H. et al. Clinical features of multiple epiphyseal dysplasia expressed in the knee. Clin Orthop Relat Res. Nov 2000(380):184-190. 
9. Anthony, S., Munk, R., Skakun, W. \& Masini, M. Multiple epiphyseal dysplasia. J Am Acad Orthop Surg. Mar 2015;23(3):164-172.

10. Koulouvaris, P., Stafylas, K., Aznaoutoglou, C., Zacharis, K. \& Xenakis, T. Isolated varus intertrochanteric osteotomy for hip dysplasia in 52 patients: long-term results. Int Orthop. Apr 2007;31(2):193-198.

11. Li, L. Y., Zhao, Q., Ji, S. J., Zhang, L. J. \& Li, Q. W. Clinical features and treatment of the hip in multiple epiphyseal dysplasia in childhood. Orthopedics. May 18 2011;34(5):352.

12. Turgeon, T. R., Phillips, W., Kantor, S. R. \& Santore, R. F. The role of acetabular and femoral osteotomies in reconstructive surgery of the hip: 2005 and beyond. Clin Orthop Relat Res. Dec 2005;441:188-199.

13. Wyles, C. C. et al. Natural History of the Dysplastic Hip Following Modern Periacetabular Osteotomy. J Bone Joint Surg Am. May 15 2019;101(10):932-938.

14. Sponer, P., Karpas, K. \& Cenĕk, J. Surgical treatment of multiple epiphyseal dysplasia in the hip joints in childhood-short-term results. Acta Chir Orthop Traumatol Cech. 2003;70(4):243-247.

15. Shibata, K. R., Matsuda, S. \& Safran, M. R. Open treatment of dysplasia-other than PAO: does it have to be a PAO? J Hip Preserv Surg. Jul 2017;4(2):131-144.

16. Miller, M. L., Clohisy, J. C., Pashos, G. E., Berglund, L. M. \& Schoenecker, P. L. Severe Hip Dysplasia in Skeletally Mature Patients With Spastic Cerebral Palsy: The Technique and Early Outcome of Comprehensive Surgical Correction (Including the Bernese PAO). J Pediatr Orthop. Jan 2021;41(1):e7-e13.

\section{Figures}




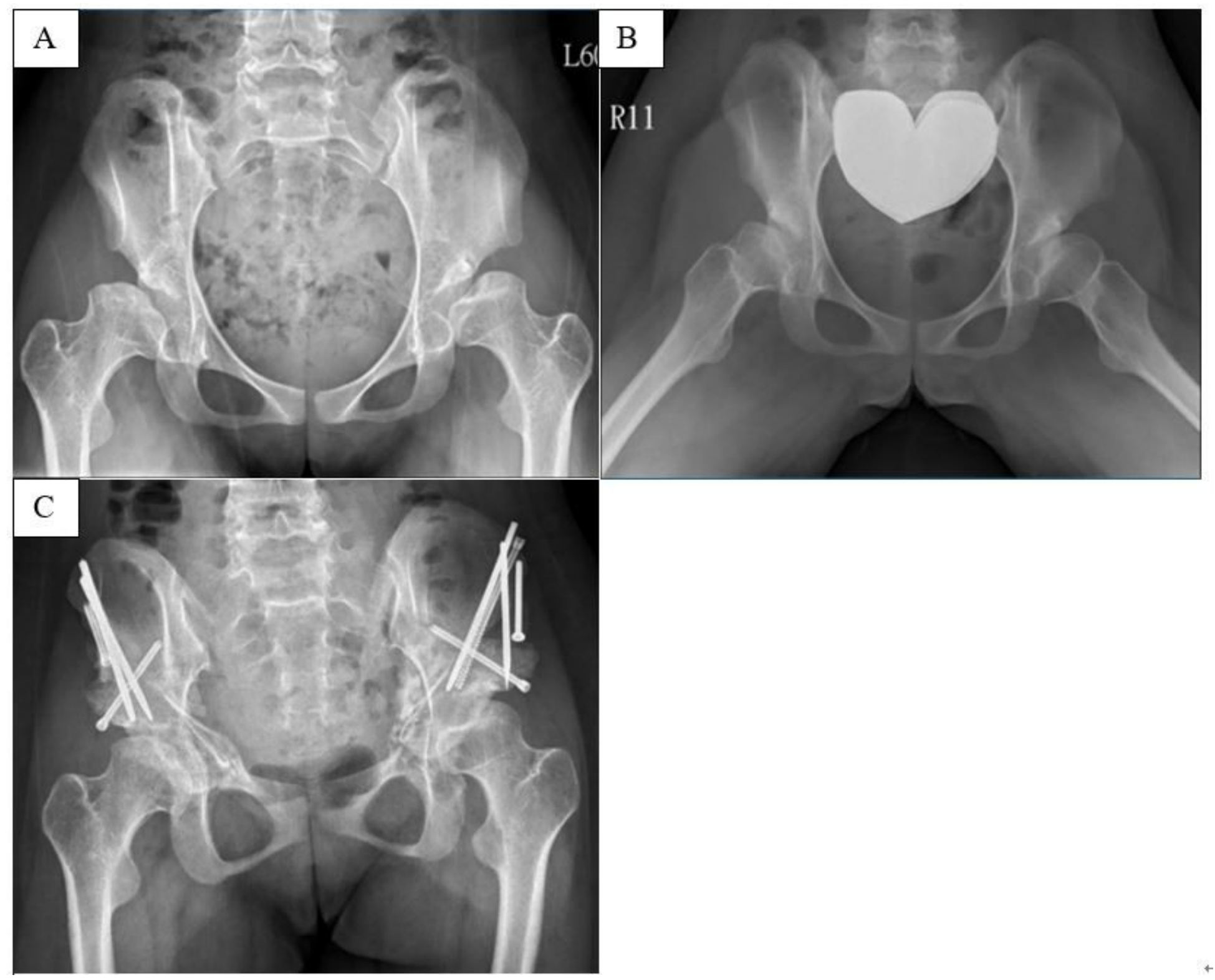

Figure 1

Panel A and B: A 15-year-old girl with multiple epiphyseal dysplasia. She complained of bilateral hip pain and limp for years. Anterior-posterior pelvic radiograph (panel A) showed severe bilateral hip dysplasia and hip subluxation with broken Shenton line. Frog leg radiograph (panel B) showed hip joints were reducible without hinge abduction with the hip in flexion and internal rotation. Panel C: She received bilateral staged Ganz PAO. Postoperative Anterior-posterior pelvic radiograph (panel C) showed correction of hip dysplasia and the femoral head was reduced.

\section{Supplementary Files}

This is a list of supplementary files associated with this preprint. Click to download.

- Table1.jpg 
- Table2.jpg

Page $11 / 11$ 